\title{
Algunas reflexiones sobre la historia de los intelectuales/diplomáticos latinoamericanos en los siglos XIX y XX \\ Introducción al dossier Intelectuales y diplomacia en América Latina
}

Carlos Marichal*

Alexandra Pita**

\section{RESUMEN}

Tanto en el siglo XIX como en el XX los intelectuales latinoamericanos han tenido protagonismo como representantes diplomáticos, favoreciendo la proyección y el prestigio cultural de sus respectivos países en ámbitos internacionales. Sin embargo, la explicación de por qué los intelectuales se involucraron en la diplomacia se ha restringido a asumir que en tanto eran representantes de la alta cultura de sus países los Estados los utilizaron para proyectar una buena imagen en el exterior. Esto dificulta la posibilidad de plantearse desde una reflexión más amplia por qué aparecieron estas figuras polivalentes y qué características tenían en momentos históricos determinados. En suma, se requiere ampliar la manera de estudiar las relaciones internacionales como un ámbito de la política exterior y dedicar más atención a las ideas, su circulación y las redes. Para ello, el presente ensayo reflexiona

Profesor de tiempo completo de El Colegio de México, correo electrónico: cmari@colmex.mx

** Profesora de tiempo completo de la Universidad de Colima, correo electrónico: apitag@ucol.mx 
sobre varias generaciones de intelectuales/diplomáticos latinoamericanos ubicándolos en una temporalidad que va desde inicios del siglo XIX a la tercera década del siglo Xx. Se enfatiza el eje Londres-París-Washington para entender las coyunturas, escenarios y actores clave en el desenvolvimiento de la diplomacia latinoamericana y sus expresiones en las esferas culturales e intelectuales.

Palabras clave: Panamericanismo, intelectuales, diplomáticos, América Latina.

\title{
Some reflections on the history of Latin American intellectuals/ diplomats in the 19th and 20th centuries
}

\begin{abstract}
Both in the nineteenth century and in the twentieth century, Latin American intellectuals have played a leading role as diplomatic representatives, favoring the projection and cultural prestige of their rights in the international arena. However, the explanation of why intellectuals got involved in diplomacy was restricted to the representatives of the high culture of the countries, and also to the United States for a good image abroad. This is the possibility of seeing beyond people to offer a broader reflection. This requires changing the way of studying international relations as the field of foreign policy to present attention to ideas, their circulation and networks. For this, this essay reflects on the Latin American intellectuals / diplomats, placing us in a temporality that goes from the beginning of the nineteenth century to the third decade of the twentieth century. The London-ParisWashington axis is analyzed to understand the conjunctures, scenarios and actors that revolve around Latin American diplomacy.
\end{abstract}

Key words: Panamericanism, intellectuals, diplomats, Latin America.

\section{INTRODUCCIÓN}

— $\mathrm{n}$ numerosos momentos de la historia moderna de Latinoamérica, ha 1 sido notable el protagonismo de intelectuales como representantes diplomáticos, hecho que ha favorecido la proyección y el prestigio cultural de sus respectivos países en ámbitos internacionales. Tanto en el siglo XIX como en el XX, numerosos escritores, ensayistas, historiadores y novelistas llegaron a ocupar cargos en las legaciones en Europa, los Estados Unidos y en la propia región. Al mismo tiempo, debe reconocerse que los cargos de diplomáticos habitualmente requerían de talentos de escritura que se aproximaban, en ocasiones, a la de los literatos. En dichas circunstancias, sería factible hablar de las funciones polivalentes del "intelectual/diplomático". 
El presente dossier ofrece una aproximación histórica, de tipo panorámica, para repensar el papel de diversas generaciones de intelectuales y escritores latinoamericanos en el ejercicio de funciones como diplomáticos a lo largo de distintas coyunturas de los últimos dos siglos, específicamente, desde las independencias, a inicios del siglo XIX, hasta mediados del siglo XX, cuando tras la finalización de la Segunda Guerra Mundial se generó (junto con la profesionalización del servicio exterior) una serie de importantes cambios que requerirían adicionales investigaciones sobre la temática de este ensayo.

La particularidad del tipo de personajes polivalentes que analizamos ha sido mencionada en numerosos trabajos que desde la historia diplomática han hecho referencia a la cualidad de algunos diplomáticos que al ser reconocidos escritores ocuparon cargos oficiales en la política exterior de su país. Si bien este tipo de aproximación contribuyó a que los historiadores pusieran atención al marco cultural que se encontraba presente en las relaciones internacionales, su explicación no deja de ser parcial. Es decir, la explicación de por qué los intelectuales se involucraron en la diplomacia se ha restringido a asumir que eran representantes de la alta cultura de sus países y que, por lo tanto, los Estados los utilizaron para dar una buena imagen en el exterior. Esto resta la posibilidad de plantearse desde una reflexión más amplia el por qué aparecieron este tipo de figuras polivalentes y qué características tenían en momentos históricos determinados. Menos aún se ha avanzado en las sutilezas que implica pensar la cuestión del orden en el término al definirse indistintamente como intelectual/diplomático o diplomático/intelectual. Esto requiere repensar el ámbito de la historia cultural de las relaciones internacionales como un cruce de caminos entre historiadores, internacionalistas, politólogos y sociólogos, para problematizar el análisis del sujeto de estudio a través de un enfoque más complejo. En buena medida, esto explica el surgimiento de la historia cultural de las relaciones internacionales, la cual intenta renovar la historia diplomática al sumar la dimensión cultural. ${ }^{1}$

1 La dimensión cultural tomó impulso en el estudio de las relaciones internacionales desde la década de 1930 cuando Pierre Renouvin llamó la atención sobre la necesidad de vincular las relaciones políticas, diplomáticas y militares entre los Estados a través de las "fuerzas profundas". Desde la década de 1960 otras interpretaciones cuestionaron el vínculo establecido desde el enfoque teórico del realismo, el cual estudiaba las actividades diplomáticas como una estrategia asociada a la política militar. Pero fue a inicios de la década de 1990 cuando esta dimensión cultural tomó realmente fuerza de la mano del concepto de soft power, acuñado por Joseph Nye. Este autor sugirió que el poder no debía ser considerado exclusivamente en términos militares y económicos (los cuales sin duda seguían conservando su estatus de high politics) sino que también debía de considerarse otro tipo de poder (low politics) que alcanzaba sus resultados no por el uso de la coerción sino de la cooptación. Los estados modificaban preferencias sin utilizar la fuerza militar sino a través de la cultura y los 
En este sentido, el concepto de diplomacia cultural implica entenderla no sólo en función de la expresión de ciertos valores nacionales y culturales dentro y fuera de un país, sino también otorgar peso a la información, los contactos personales, los programas culturales y todo aquello intangible que requiera de una pluralidad de herramientas para persuadir, captar y convencer. Implica considerar juicios, valores y normas de un gran número de actores sociales encabezados por el Estado y prestar una atención especial a los diplomáticos como actores principales. Según Juliette Dumont, la diplomacia cultural implica la utilización de relaciones específicas para llevar a cabo objetivos no sólo culturales, sino también políticos, comerciales o económicos. $^{2}$

Como ha planteado Juan Pablo Scarfi en un lúcido ensayo, desde la historia intelectual, cultural y trasnacional se ha generado en los últimos años nuevos planteamientos sobre el papel de la cultura en la diplomacia. Esto se encuentra vinculado con la manera en que los intelectuales participaron del proceso de formación de una burocracia internacional para los estados (entre 1880 y 1946 cuando se moderniza la diplomacia y se organiza la estructura de ministerios, embajadas, consulados). ${ }^{3}$

Como se podrá observar en las siguientes páginas de este ensayo que sirve a manera de introducción del dossier, el pensar a los intelectuales/diplomáticos latinoamericanos requiere identificar coyunturas, escenarios y actores. De inicio, requiere ubicar los temas y problemas a considerar en el tiempo y el espacio concretos. Sin miras a fomentar un nacionalismo reiterativo, es evidente que esta reflexión histórica tiene que partir de las características de los orígenes de la diplomacia de los diferentes países latinoamericanos desde que inician los procesos de insurgencia, no sólo por las acciones extraoficiales de las facciones para conseguir armas, sino también porque era necesario dar legitimidad a su proyecto de nación a partir del reconocimiento diplomático de los otros países, en especial, europeos.

Así, no se trata sólo de entender por qué en el conflictivo inicio del siglo XIX miraron al mundo atlántico en busca de reconocimiento (y por ende, apoyo) para conformarse como naciones. Y para ello es importante trazar un recorrido que se fija en el complejo eje de Londres-París-Washington. Como veremos en las siguientes páginas, estas ciudades fueron claves para la diplomacia latinoamericana durante el siglo XIX y hasta la tercera década del siglo XX. Si las dos primeras ciudades son escenarios tradicionales de la

valores como bienes intangibles al servicio de los objetivos de un país. Pita, "La diplomacia cultural mexicana en la entreguerras”, pp. 107-108.

2 Dumont, L'Institute et le Brésil, p. 19.

3 Scarfi, “Introducción”, <http://historiapolitica.com/dossiers/diplomacia-circulacion-saberes/>. 
diplomacia europea, la tercera anuncia el avance de Estados Unidos y su rápida expansión durante la segunda mitad del siglo XIX. Este desplazamiento geográfico refleja el camino seguido por la política y la economía, y también sugiere la importancia de analizar los cambios en las formas de hacer diplomacia en la primera mitad del siglo $\mathrm{Xx}$, los cuales siguieron teniendo un fuerte contenido nacional y bilateral, aunque también fueron incorporando la participación muy activa en organismos internacionales incluyendo, por ejemplo, las conferencias Panamericanas y la Sociedad de Naciones, mismas que reúnen a sus participantes en distintas conferencias internacionales de gran interés para la historia cultural e intelectual de Latinoamérica.

\section{LONDRES EN LA DÉCADA DE 1820: ESCRITORES Y DIPLOMÁTICOS LATINOAMERICANOS EN LA ÉPOCA DE INDEPENDENCIA}

El arranque de las guerras de independencia fue clave en la aparición de esa figura novel en la historia latinoamericana que era el diplomático o ministro plenipotenciario, aunque inicialmente esta función no se limitaba a un encargo oficial, sino que se asemejaban a otra categoría de personalidades que han sido bautizados como "letrados patriotas". ${ }^{4}$ Nos referimos al hecho de que desde el inicio de las primeras luchas por la independencia podemos identificar a intelectuales latinoamericanos militantes que viajaron fuera de la región por motivos de persecución política para difundir el primer republicanismo o con objeto de obtener apoyos concretos de tipo financiero y armas para apuntalar la insurgencia. La historiografía reciente, que ha dado seguimiento a estas figuras, argumenta que un número selecto de tempranos patriotas fueron actores importantes en dos de las cunas más significativas del liberalismo decimonónico europeo y americano, concretamente en el Cádiz de las Cortes en los años de 1810-1813, así como en Filadelfia, especialmente en los años de 1812-1820. Como ha señalado Rafael Rojas, debe ahondarse en esta historia de los primeros intelectuales republicanos latinoamericanos en dichas ciudades, ya que allí desplegaron una fértil actuación tanto en su prédica política como en sus labores como escritores, propagandistas y editores. ${ }^{5}$

Pero cabe señalar también la importancia de fijar atención en la ciudad de Londres, la cual pronto cobró especial protagonismo como escenario clave de

4 Myers "El letrado patriota: los hombres de letras hispanoamericanos en la encrucijada del colapso del imperio español en América”, pp. 121-144.

5 Rojas, "Traductores de la libertad; el americanismo de los primeros republicanos", pp. 205-226. 
negociaciones internacionales realizadas por tempranos dirigentes e ideólogos de las emergentes naciones americanas que pugnaban por obtener el reconocimiento de su soberanía. En este fascinante y complejo proceso tuvo una participación destacada una serie de diplomáticos/intelectuales latinoamericanos, especialmente en los años de 1822-1825 cuando se establecieron las primeras legaciones en la capital inglesa por parte de los nuevos gobiernos de México, Perú, Gran Colombia, Chile, Provincias Unidas del Río de la Plata, la República Federal de Centroamérica y del Imperio de Brasil. ${ }^{6}$

Existían algunos antecedentes de acercamiento con Gran Bretaña desde el arranque mismo de las luchas de la insurgencia. Los primeros representantes en llegar a Londres a pregonar la futura independencia consistieron en una comisión nombrada por la Junta de Caracas que incluía a Simón Bolívar, Luis López Méndez y Andrés Bello, quienes llegaron a la capital británica en junio de 1810 con objeto de lograr un apoyo militar para apuntalar el novísimo proceso de liberación de Venezuela. Las autoridades británicas no quisieron recibirlos formalmente ya que en esos momentos Gran Bretaña apoyaba la lucha de los patriotas españoles en contra de Napoleón. Por ello, Bolívar no tardó en regresar a Caracas para seguir con la insurrección militar y política contra el régimen español mientras que López Méndez se quedó en Londres para convertirse en permanente agente y agitador político; por su parte, Andrés Bello se concentró en sus labores de escritor aprovechando la magnífica biblioteca de su compatriota, el legendario Francisco Miranda, quien desde 1804 vivía en la capital inglesa. Tiempo después Bello ocupó brevemente un cargo como bibliotecario de José Blanco White, escritor español exiliado y protegido de Lord Holland, quien fue mecenas de tantos liberales españoles e hispanoamericanos en la época. Bello también mantuvo estrecha relación con el gran intelectual y polemista mexicano, Fray Servando Teresa de Mier, residente en Londres, quien colaboraba estrechamente con Blanco White en la edición del legendario periódico liberal El Español.

En junio de 1820 el vicepresidente de Gran Colombia, Francisco Antonio Zea, llegó a Londres como ministro plenipotenciario con poderes para buscar financiamiento para los ejércitos de Bolívar, así como para renegociar deudas pendientes de pago con proveedores de armas británicas. Zea fue el primer representante diplomático oficial de una nación latinoamericana, aunque el Secretario de Asuntos Exteriores británico, Castlereagh, rehusó recibirle. Pese a ello, Zea comenzó a negociar con diversas casas financieras londinenses y en 1822 logró la contratación de un primer empréstito para el gobierno de Gran Colombia por unos 3 millones de libras esterlinas. Este

6 Uno de los más importantes libros sobre este tema es el estudio clásico de Rodríguez O., El nacimiento de Hispanoamérica: Vicente Rocafuerte y el hispanoamericanismo, 1808-1832. 
éxito fue tan sonado que instó a otros próceres latinoamericanos a gestionar préstamos para financiar sus ejércitos comprometidos en las últimas etapas de la lucha por la independencia. El brigadier y "director supremo" del gobierno chileno, Bernardo O’Higgins, por ejemplo, confió en los servicios del político y escritor, Antonio José de Irisarri, quien llegó a Londres en 1820 y pronto se puso a negociar un empréstito para el gobierno chileno (en 1822) en este caso con la casa financiera de Hullett Brothers, si bien Irisarri luego utilizó sus contactos para llevar a cabo especulaciones en la Bolsa de Londres, incluyendo inversiones en una importante cadena de compañías mineras de plata, las cuales se hundieron en 1827. ${ }^{7}$ Andrés Bello se benefició de su amistad con Irisarri, y por ello desde 1822 fue nombrado secretario de la Legación de Chile, lo cual le sacó de apuros financieros hasta su posterior partida a Santiago de Chile en 1829, donde habría de convertirse en uno de los intelectuales más destacados y fundador de la Universidad de Chile en 1842.

En los años de 1823-1825 llegaron a Londres otros diplomáticos distinguidos que no sólo eran figuras intelectuales y políticas conocidas, sino además activos agentes financieros para sus respectivos países. Entre ellos destacaban José María Hurtado, embajador de Colombia; Vicente Rocafuerte y José Michelena, representantes de México, Juan García del Río, delegado de Perú, y Mariano Egaña, de Chile. ${ }^{8}$ Desde el punto de vista del presente ensayo, lo que interesa destacar fue el papel de este grupo de tempranos diplomáticos latinoamericanos como escritores, directivos de periódicos y de colecciones editoriales en Londres en esos años. Irisarri, por ejemplo, editó en Londres en 1820, el periódico El Censor Americano, que abogaba claramente por el establecimiento de algún tipo de monarquía constitucional en las tierras hispanoamericanas. Por su parte, Andrés Bello se dedicó con pasión a una gran labor editorial, incluyendo la publicación de la revista Biblioteca Americana en la capital británica en 1823, y luego de la revista El Repertorio Americano en 1826 y 1827. En ambos casos contó con apoyos financieros de la legación de México, y más particularmente de Vicente Rocafuerte, quien fue pieza clave en fomentar un ambiente prolífico de publicaciones y reuniones tanto entre los diplomáticos e intelectuales latinoa-

7 “Aunque guatemalteco por nacimiento, Antonio José de Irisarri [1786-1868] era el segundo ministro enviado por Chile a Gran Bretaña. Siguiendo instrucciones explícitas, pero no escritas de Bernardo O’Higgins, Irisarri contrató un empréstito de un millón de libras a través de la casa londinense de Hullett Brothers. Cuando la noticia llego (sic) a Chile, el gobierno reacciono (sic) en contra, pero era demasiado tarde... Los bonos chilenos ya se vendían en el mercado de Londres desde hacía algunos meses”: Veliz, “Egaña”, pp. 637-638.

8 Información sobre estos diplomáticos puede encontrarse en Jaksic, Andrés Bello: Scholarship and Nation-Building in Nineteenth-Century Latin America. También de gran utilidad son los ensayos editados por Lynch, Bello y Londres. 
mericanos como entre los numerosos exiliados liberales españoles que residieron en Londres entre 1823 y 1833, experiencia fascinante descrita en el estudio clásico de Vicente Llorens, Liberales y Románticos. ${ }^{9}$

La efervescencia que caracterizó la actividad de la primera generación de diplomáticos latinoamericanos en Londres en los años de 1823-1826 estuvo ligada a varios factores, incluyendo la firma de los primeros tratados de comercio libre con Gran Bretaña, las campañas por promover el reconocimiento de las nuevas repúblicas y la obtención de fondos financieros de apoyo. Ya hemos indicado que los primeros dos gobiernos latinoamericanos en firmar contratos para un empréstito extranjero fueron los de Colombia y Chile, pero para 1825 la mayoría de los flamantes Estados también habían logrado emitir bonos en el mercado londinense y ya acumulaban cuantiosas deudas externas, mismas que prometían pagar con los ingresos aduaneros de sus gobiernos. Los bonos de Argentina, Brasil, la Federación Centroamericana, Chile, Gran Colombia, México y Perú eran vendidos y comprados a precios elevados en la Bolsa de Londres, y el furor por esos valores exóticos, pero lucrativos siguió durante un par de años. El bullicio en la bolsa de Londres y el éxito de las operaciones financieras permitieron una intensa actividad diplomática, política y cultural por parte de las legaciones en la capital británica, pero la coyuntura favorable no fue duradera. La crisis financiera que se desató en los mercados financieros británicos desde diciembre de 1825 provocó el derrumbe de los valores de los bonos latinoamericanos y pronto llevó a moratorias por parte de todos los gobiernos latinoamericanos, salvo el de Brasil. ${ }^{10}$ Como consecuencia, la mayoría de las legaciones se encontraron en grandes estrecheces económicas. La partida de Bello hacia Chile, a principios de 1829, al igual que la de Rocafuerte para México, eran sintomáticas; a su vez, la moratoria chilena de 1826 incidió en la bancarrota personal de Irissari, quien pronto regresaría a su tierra natal de Guatemala. En resumidas cuentas, los tempranos años dorados de los primeros diplomáticos/intelectuales de Latinoamérica en la capital inglesa luego darían paso a una época mucho menos brillante e intelectualmente menos productiva.

\section{PARÍS A MEDIADOS DEL SIGLO XIX: DIPLOMACIA, POLÍTICAY ESCRITURA}

Si Londres puede considerarse el eje de más intensa actividad diplomática latinoamericana en la última etapa de las guerras de independencia, en dece-

9 Llorens, Liberales y románticos: una emigración española en Inglaterra, 1823-1834.

10 Para detalles véase Marichal, Historia de la deuda externa de América Latina, caps. 1 y 2. 
nios posteriores se observaron cambios significativos en la dinámica de las relaciones exteriores de los países de la región. Concretamente, a partir de la guerra e invasión de los Estados Unidos contra México entre 1846 y 1848, París se convirtió rápidamente en la ciudad que atrajo a mayor número de talentosos o diplomáticos, así como de exiliados latinoamericanos, quienes encontraron en la ciudad luz una plataforma idónea para promover la defensa de los intereses hispanoamericanos en Europa. Los exiliados y los diplomáticos que referimos rechazaron numerosos casos de intervencionismo y expansionismo colonialista de potencias extranjeras en diferentes tierras de América Latina y el Caribe. ${ }^{11}$ Fue especialmente notorio el caso de la expedición del aventurero militar norteamericano, William Walker, en Nicaragua en 1854, la cual amenazó al conjunto de estados centroamericanos. Un decenio más tarde, la mayoría de los representantes latinoamericanos en Europa se opusieron a las políticas agresivas del gabinete español de la Unión Liberal, el cual promovió varias intervenciones militares, incluyendo la ocupación militar de Santo Domingo en 1861, seguida por la guerra naval con Perú en 1865 y la ocupación de las islas Chincha, mejor conocidas como las islas del guano. Sin embargo, la expresión más violenta del imperialismo europeo fue la invasión y ocupación de la mayor parte del territorio de México entre 1862 y 1867 por el ejército francés, a pesar de la resistencia del gobierno de Benito Juárez y de sus protestas internacionales.

Como ha argumentado concienzudamente Arturo Ardao, fue el intelectual colombiano José María Torres Caicedo, radicado en París desde 1853, ${ }^{12}$ quien llevó a cabo la más amplia y constante labor como periodista y publicista a favor de la causa de la unión latinoamericana en la capital francesa.13 Torres Caicedo era un diplomático polivalente —algo bastante común en la

11 Marichal, "El nacimiento de los estudios internacionales sobre América Latina: comentarios a las obras de José María Torres Caicedo y Carlos Calvo a mediados del siglo XIX”.

12 Desde muy joven, José María Torres Caicedo (1830-1889) escribió en varios periódicos radicales en Bogotá en 1849 y 1850, pero pronto tuvo que emigrar para recuperarse de una herida sufrida en un duelo, al parecer por razones políticas. Se pasó los años de 1851 y 1852 en Nueva York hasta recalar en París donde viviría la mayor parte del resto de su vida: Ardao, América Latina y la Latinidad, p. 59.

13 Entre sus obras se destacó las bases para la formación de una liga latinoamericana, la cual se escribió en 1861, se reprodujo en 1865 y en 1872 bajo el título "Unión Latino Americana. Pensamiento de Bolívar para formar una liga americana, su origen y sus desarrollos”. En éste se dedicó a desarrollar un plan de confederación de los estados latinoamericanos, entendiendo que este sistema era el más conveniente como reunión de estados soberanos que buscaban resguardar los derechos territoriales y tradiciones de raza (legua, religión, costumbres, aspiraciones comunes), ante los peligros externos que pongan en peligro su independencia. Sobre este personaje y su obra véase Granados, "Congresos e intelectuales en los inicios de un proyecto y de una conciencia continental latinoamericana”, pp. 56-61. 
época - siendo ministro plenipotenciario de Colombia en París, Londres y la Santa Sede en los años de 1850, pero luego también de Venezuela, con representación en París y Bruselas, y unos años más tarde de El Salvador. Esta polivalencia era bastante común debido al escaso desarrollo de la carrera diplomática (y los escasos recursos pecuniarios de las legaciones) de la mayoría de los países latinoamericanos y, quizá por ello no era extraño que se eligieran como representantes a escritores de nota o políticos bien conectados para ocupar los cargos de ministros plenipotenciarios tanto por sus conexiones, su capacidad de sostenerse con escritos en revistas y la prensa, así como por su manejo de idiomas.

Al mismo tiempo, es conveniente notar que una de las características de las labores de los diplomáticos latinoamericanos en Europa a mediados del siglo XIX era que no requerían una dedicación tiempo completo y, por lo tanto, disponían de bastantes horas libres para escribir. Torres Caicedo, quien ganaba muy poco como representante de Venezuela, pudo destinar mucho tiempo a redactar artículos y ensayos en la prensa europea: desde 1857, y durante muchos años, contribuyó a El Correo de Ultramar (18421886), periódico literario y político que se publicaba dos veces al mes en París y que había sido fundado por un rico comerciante francés, publicación que logró crear una amplia red de suscripciones tanto en las Antillas como en la América española. El atractivo de este periódico consistía en que ofrecía a sus lectores una combinación de notas políticas con numerosas noticias sobre la moda y la actividad cultural y científica en París, así como artículos literarios. Contó, sobre todo, con plumas de intelectuales, políticos y artistas españoles como Eugenio de Hartzenbusch, Mariano Urrabieta, Eugenio de Ochoa, Emilio Castelar, Manuel Fernández y González, pero también con algunos hispanoamericanos. ${ }^{14}$ Torres Caicedo, quien pasó a ser su director en 1857, cuidó mucho la relación con España y estableció estrechos vínculos con el intelectual gaditano, Eduardo Asquerino, editor del periódico madrileño La América (1857-1886). Desde su fundación, éste fue otro importante vehículo político y literario, señalado por Ardao como la publicación periódica de su tipo de mayor difusión en el mundo de lengua española, con suscripciones en España, gran parte de la América española (incluyendo veinte agencias de suscripción en México) y ciertas ciudades de los Estados Unidos. De hecho, Torres Caicedo publicó un artículo en La América en 1858

14 Sablonierre, "El Correo de Ultramar (1842-1886) y la ciencia: entre labor educativa y propaganda política". 
en el cual usó la expresión “América Latina”, primicia en los medios madrileños. ${ }^{15}$

En junio de 1861 los diplomáticos hispanoamericanos acreditados en París y Londres le dirigieron a Torres Caicedo una nota destinada a reconocer sus aportaciones en reconocimiento de esta amplia labor como publicista y editor entusiasta de la defensa de la cultura y de la soberanía de las repúblicas hispanoamericanas. Decía el documento:

\begin{abstract}
Es a usted, Señor, a quien se debe haber levantado el glorioso pendón de los Estados Hispano-Americanos; usted en periódicos españoles y franceses ha defendido los derechos soberanos de esas Repúblicas, siempre que algunas Naciones poderosas han pretendido desconocer la justicia que a ellas asistía. Siga usted en su obra filantrópica, en la cual trabaja desde hace ocho años con tanto celo como desinterés y obtendrá los beneficios de todos cuantos rinden culto a lo bello, lo bueno y lo grande... ${ }^{16}$
\end{abstract}

Firmaron como afectísimos "compatriotas" un conjunto distinguido, pero algo variopinto de latinoamericanos que incluía a Pedro Gálvez, ministro plenipotenciario de Perú; Carlos Calvo, encargado de negocios del Paraguay; Juan Bautista Alberdi, ministro plenipotenciario de la Confederación Argentina; Andrés Santa Cruz, antiguo protector de la Confederación PerúBoliviana y ministro plenipotenciario de Bolivia; Víctor Herrán, ministro plenipotenciario de Honduras y de El Salvador, así como los ministros de la Confederación Granadina, Guatemala, Ecuador y Venezuela.

Se trataba de un verdadero club de diplomáticos, pero es interesante notar que entre ellos se contaban tanto escritores y publicistas destacados, además de veteranos políticos. Juan Bautista Alberdi, probablemente el autor latinoamericano más prolífico de la época, escribió constantemente durante tres decenios en periódicos de París y de América, a lo cual se agregaba su profusa producción de folletos y tratados políticos, legales y económicos. Gran parte de sus textos fueron redactados durante su larga estancia en París, (1854-1880) y se han convertido en clásicos del periodismo y de la ciencia política latinoamericana.

Conviene resaltar, en este sentido, la importancia de París en esta época como el punto más importante de exportación de libros y revistas a los países latinoamericanos, tal como lo demuestra el considerable número de libreros/editores que se dedicaban a este negocio en la Ciudad Luz, incluyendo la

15 Ardao, América Latina y la latinidad, pp. 211-217; véase también López-Ocón, Biografía de "La América": una crónica hispano-americana del liberalismo democrático español (1857-1886).

16 Ardao, América Latina y la latinidad, pp. 81-82. 
exportación de textos en francés, pero también gran número de traducciones al español. En los años de 1860 algunas de las librerías parisinas que además figuraban como editoras, y que publicaban numerosos textos para el público español y latinoamericano (en francés y español) incluían las de A. Durand, Garnier Hermanos, y Hachette y Cia. y tenían estrechas relaciones con la librería Bailly-Baillière en Madrid que también reexportaba libros y prensa a diversas ciudades latinoamericanas. Algunas de estas empresas evolucionarían más tarde hasta constituirse como editoriales con cierta especialización temática.

Uno de los más activos propulsores de la unidad latinoamericana en Europa en esta época era el diplomático peruano Pedro Gálvez Egúsquiza, con quien estrecharon lazos Torres Caicedo y Carlos Calvo. El gobierno de Perú, encabezado por el general Ramón Castilla, estaba empeñado en estos años en defender los principios de autodeterminación y de no intervención. En un acto de solidaridad con países amigos, Castilla había nombrado en 1855 a Pedro Gálvez como ministro plenipotenciario de Perú, pero también representante oficioso para los países de la América Central, Nueva Granada y Venezuela, cumpliendo un importante papel en el respaldo a la defensa de Costa Rica a raíz de las amenazas que provocó la invasión de Nicaragua por los filibusteros de William Walker. También obtuvo la adhesión a su política americanista de los gobiernos de El Salvador y Guatemala, naciones puestas en riesgo por la ambigua posición de las autoridades estadounidenses. Castilla y Gálvez ejercieron un papel clave en la redacción y firma del Tratado Continental firmado en Santiago de Chile el 15 de septiembre de 1856 entre Perú, Ecuador y Chile para asegurar la soberanía y la unidad política y comercial de los estados americanos.

En 1859 Gálvez fue nombrado ministro plenipotenciario del Perú en Francia, y en 1860 asumió también la tarea de representar Perú ante el gobierno español, aunque siguió residiendo en París. Es interesante notar que en los archivos histórico/diplomáticos del Perú se encuentra una nota de esta época de José María Torres Caicedo al Ministro de Relaciones Exteriores de Perú con una solicitud suya para que el gobierno peruano auspiciara la publicación de un periódico para la defensa de los derechos e intereses de las repúblicas Hispano Americanas (1860). ${ }^{17}$ La razón era sencilla: el gobierno peruano era entonces el más rico de toda América Latina gracias a los ingentes ingresos provenientes de las exportaciones del guano, recurso que era propiedad del Estado, si bien era comercializado por empresas privadas.

17 En catálogo en línea del Archivo histórico central del Ministerio de Relaciones Exteriores de Perú, documento 28 de la Correspondencia diplomático del año de 1860. $<$ http://www.rree.gob.pe/portal/ArchivoCentral/Catalogo/ACInventario.nsf/> 
Como puede observarse en otra correspondencia diplomática que está registrada en los archivos diplomáticos peruanos, hacia mediados del siglo XIX, el gobierno de Ramón Castilla acostumbraba financiar artículos en periódicos en Europa con objeto de promover una imagen positiva para el país y sus productos de exportación. ${ }^{18}$ Es éste un tema que merece ser explorado en mayor detalle a nivel comparativo, ya que la mayoría de los gobiernos latinoamericanos siguieron el ejemplo peruano en épocas posteriores, destinando, en ocasiones, sumas importantes para financiar artículos que pudieran redundar en más inversiones, comercio o inmigración.

El gobierno de Paraguay también propuso impulsar la defensa de su soberanía al tiempo que propiciaba una propaganda intelectual a favor del conjunto de la región. En enero de 1860 el general y presidente, Carlos Solano López, nombró al abogado y escritor argentino, Carlos Calvo "Encargado de Negocios del Paraguay en Londres y París” por un período de dos años prorrogables, con un sueldo de 6,000 de pesos anuales. Calvo aceptó inmediatamente y salió para Londres con objeto de llevar a cabo las negociaciones para intentar restablecer relaciones entre Paraguay y Gran Bretaña, si bien tuvo que resolver previamente una serie de conflictos pendientes entre los dos países. Al principio, no tuvo éxito ya que el secretario de asuntos exteriores de Inglaterra, Lord John Russell, se negó a recibirlo, acusando a Paraguay de haber agredido a ciudadanos ingleses y de provocar conflictos entre un barco inglés y uno de la Armada paraguaya. Eventualmente, Calvo tuvo la habilidad de lograr "la intervención oficiosa del señor Thouvenal, ministro de Negocios Extranjeros de Francia, y del Conde de Flahaut, embajador de Francia en Londres”, y muy amigo de Russell. ${ }^{19}$ Con ello se fueron restableciendo las relaciones diplomáticas entre Paraguay y Gran Bretaña. ${ }^{20}$

Después de dejar su puesto de ministro plenipotenciario para Paraguay en 1863, Calvo se trasladó a París y se dedicó esencialmente a escribir y dar clases de derecho internacional, obteniendo un gran reconocimiento por ello en muchos círculos académicos y diplomáticos europeos. Entre varios proyectos editoriales ambiciosos que Calvo impulsó en la Ciudad Luz, se incluyó una serie de tempranas obras sobre relaciones internacionales latinoame-

18 El portal del Ministerio de Relaciones Exteriores del gobierno peruano es una fuente de gran interés para los historiadores. Véase en <http://www.rree.gob.pe/portal/archivos>.

19 Limantour, "Memoria sobre la vida...”, p. 10. Paradójicamente, debe notarse que precisamente en esta época Flahuat y Russell estaban en estrecha relación secreta, planeando la intervención militar en México.

20 Para un recuento pormenorizado de las negociaciones emprendidas debe consultarse el texto autobiográfico de Carlos Calvo publicado en 1864 en París por la Librarie A. Durand, titulado Una página de derecho internacional o la América del Sur ante la ciencia del derecho de gentes moderno. 
ricanas, por ejemplo, su Colección... de los tratados... de la América Latina, así como de sus Anales históricos de la revolución de la América Latina... de 1808 hasta el reconocimiento de la independencia, los cuales, en conjunto, sumaron 16 volúmenes. Es evidente que dicha tarea no pudo llevarse a cabo sin disponer de considerable tiempo y recursos financieros para las tareas editoriales (incluyendo el copiado y corrección) así como la impresión y la difusión de las obras. El gobierno peruano fue muy generoso en dedicar cuantiosos fondos para adquirir y difundir las primeras compilaciones sobre relaciones internacionales de América Latina que fueron editadas en París por Carlos Calvo. ${ }^{21}$ En resumidas cuentas, existen amplios testimonios de la riqueza de la labor realizada por más de una decena de diplomáticos e intelectuales en París a mediados del siglo XIX que bien pueden ser objeto de investigaciones históricas futuras mucho más detalladas.

\section{WASHINGTON: DE LA GUERRA CIVIL (1861-1865) AL PANAMERICANISMO DE INICIOS DEL SIGLO XX}

Una tercera ciudad donde convivieron buen número de diplomáticos e intelectuales latinoamericanos desde mediados del siglo XIX fue la de Washington D.C., donde hemos podido contabilizar ocho legaciones de sendos países de la región durante y después de la terrible guerra civil en ese país (18611865). Para ilustrar la trascendencia de las experiencias de los diplomáticos/intelectuales latinoamericanos en los Estados Unidos en esta dramática coyuntura comentaremos aquí algunas facetas de las misiones de tres figuras de primer orden: Domingo Faustino Sarmiento, ministro plenipotenciario de Argentina en los Estados Unidos entre 1865 y 1868; Benjamín Vicuña Mackenna, ministro de Chile entre 1865 y 1866, y Matías Romero, ministro de México desde 1861 hasta fines de 1867. En los tres casos señalados existen fuentes muy ricas para el estudio de sus gestiones oficiales - especialmente su correspondencia-, pero también son abundantes los escritos que realizaron en Nueva York y Washington en estos años.

Después de haber sido director de escuelas del Estado de Buenos Aires, Domingo Faustino Sarmiento (quizá el intelectual argentino más prolífico de su época), fue nombrado gobernador de su provincia natal de San Juan en 1862, cargo que ocupó durante algo más de dos años, llevando a cabo una labor importante en la creación de escuelas, el impulso a la minería y a una serie de reformas modernizadoras. No obstante, también tuvo que dedicar

21 Marichal, "El nacimiento de los estudios internacionales de América Latina...”, pp. 720725. 
grandes esfuerzos a la lucha política y militar regional contra Ángel Vicente Peñaloza, largo tiempo jefe de gauchos montoneros en La Rioja, San Luis y San Juan. Después de la derrota y asesinato del "Chacho Peñaloza”, en noviembre de 1863, el general Bartolomé Mitre, presidente de Argentina resolvió nombrar a Sarmiento como ministro plenipotenciario para llevar a cabo una misión de representación en los Estados Unidos. ${ }^{22}$ En realidad, este nombramiento no se hizo efectivo hasta fines de 1864, pero además de reforzar relaciones con los Estados Unidos, Mitre instruyó a Sarmiento a pasar primero por Chile y Perú, precisamente en estos años de conflictos militares en el Pacífico, a raíz de la prolongada intervención de la armada española que bombardeó los puertos de Valparaíso y Callao.

Sarmiento pasó por Santiago y Lima (donde participó en un Congreso interamericano a favor de la paz a principios de 1865), hasta llegar a Nueva York en mayo de 1865, en un momento dramático de la historia de los Estado Unidos. El ejército de la Unión finalmente había logrado vencer a las fuerzas de la Confederación tras una serie de batallas en extremo cruentas. Sin embargo, apenas cinco días después de la rendición del general Roberto Lee en Richmond, el presidente Abraham Lincoln fue asesinado el 14 de abril. Sarmiento relató sus experiencias en una fascinante correspondencia con su bien amada Aurelia Vélez, en la cual describió tanto su asistencia a las paradas militares de 200,000 tropas triunfantes de la Unión en Washington, y luego en Nueva York, así como su visita al tribunal donde se juzgaban a los cómplices del asesino de Lincoln. ${ }^{23}$

En numerosos artículos que mandaba a El Zonda de San Juan (así como en una amplia correspondencia y diversa con la gran educadora argentina Juana Manso y con Aurelia Vélez), Sarmiento ofreció descripciones muy emotivas de los Estados Unidos en esta época; describió con gran fervor sus industrias, medios de transporte de ferrocarriles y vapores, el bullicio y dinamismo de las principales ciudades, especialmente Nueva York, Boston, Filadelfia y Chicago, los magníficos paisajes, ríos y lagos, pero sobre todo, la multiplicación de las escuelas y las universidades. Todo le entusiasmaba de ese país y por ello se dedicó con pasión a escribir gran número de artícu-

22 Para correspondencia pertinente ver Sarmiento Mitre 1846-1868, Buenos Aires, Museo Mitre, 1911. Y también el volumen Cartas confidenciales a Manuel R. García-Mansilla, Buenos Aires, imprenta de Coni, 1917, que puede consultarse en versión en pdf en <http://www.biblioteca.org.ar/libros/200191.pdf>.

23 Véase esta correspondencia en Domingo Faustino Sarmiento, Obras Completas, vol. XXIX, pp. 24-27 y 30-34. 
los, editar una revista periódica (titulado Ambas Américas) y varios libros. ${ }^{24}$ Entre las primeras obras que entregó a la imprenta en Nueva York fue una biografía titulada Vida de Abraham Lincoln, texto que hizo difundir tanto en los Estados Unidos como en gran parte de América del Sur. ${ }^{25}$ Durante 1865 hasta fines de 1867, el intelectual y ministro argentino viajó por gran parte del norte y centro de los Estados Unidos con especial afán por conocer escuelas, asociaciones de maestros y maestras, y una amplia serie de universidades; de hecho, recibió varios doctorados honoris causa de universidades como las de Brown y de Michigan durante sus viajes. Todo ello le sirvió para escribir otro libro titulado Las escuelas: base de la prosperidad y de la república en los Estados Unidos, publicado en Nueva York en 1866 con un tiraje cercano a los 2 mil ejemplares, distribuidos en toda América. Claramente, esta obra le sirvió para consolidar sus planes de extender la educación popular por toda Argentina, como lo haría a partir de su elección como presidente en $1868 .^{26}$

Otro gran intelectual (ya amigo de Sarmiento) que estuvo un tiempo en Nueva York y Washington en 1865 y 1866 fue Benjamín Vicuña Mackenna, quizá el escritor más prolífico de Chile de su tiempo. ${ }^{27}$ Había sido enviado por su gobierno como agente confidencial para promover la campaña de Chile en contra de las intervenciones de España, en especial a raíz de las actividades belicosas de las fuerzas navales hispanas que patrullaban todo el Pacífico sur y habían bombardeado a los puertos de Valparaíso y de Callao, además de ocupar las isla Chincha, tan ricas en depósitos de guano.

Vicuña Mackenna llegó a Nueva York en noviembre de 1865, unos meses después de Sarmiento. Su misión consistió en trabajar con la prensa norteamericana para ganarles a la causa de Chile contra España. Ello le ganó la enemistad de William Seward, secretario del Departamento de Estado que simpatizaba con España. De todas maneras, Vicuña Mackenna trabajó de manera algo sigilosa en la compra de buques de guerra para Chile, aunque sería atacado en repetidas ocasiones por los representantes españoles que lo llevaron, inclusive, a los tribunales. Al mismo tiempo, el escritor chileno se dedicó a escribir artículos sin parar en la prensa de Nueva York y allí pudo

24 Para la edición en pdf de Ambas Américas véase la edición de Obras Completas de Sarmiento (Ambas Américas), vol. XXX, publicado por la Universidad Nacional de la Matanza, Buenos Aires, 2001, <https://archive.org/details/ambasamricasre00sarm/page/n5>.

25 Estaba tan bien escrita esta obra que inclusive hoy en día sigue llamando la atención como puede observarse en su reedición por la editora madrileña de Marcial Pons en 2017.

26 Para ver ésta y demás obras completas en formato digital pueden consultarse en el sitio web de Casa Natal de Domingo Faustino Sarmiento, en la ciudad de San Juan, los cuales pueden consultarse en el sitio en línea <https://casanatalsarmiento.cultura.gob.ar/bibliotecadigital/obras-sarmiento/>.

27 Donoso, Vicuña Mackenna: su vida, escritos y su tiempo, 1831-1886. 
editar un periódico titulado La Voz de América, durante diez meses, el cual se dedicaba a atacar el imperialismo hispano. Éste tuvo amplia difusión: de 2 mil ejemplares se enviaban mensualmente mil a Cuba, 200 a las legaciones hispanoamericanas en Washington, 100 al cónsul de Chile en París (para redistribuir en Europa) y el resto a los cónsules chilenos a la largo de las Américas. ${ }^{28} \mathrm{Al}$ mismo tiempo, como su amigo Sarmiento, dedicó buena parte de su tiempo a escribir una serie de folletos y un importante libro titulado Chili, Spain and the United States, publicado en Nueva York en 1866 con el fin de defender a su país, distribuyendo 300 ejemplares a miembros del Congreso de los Estados Unidos, 400 a los principales periódicos y los demás en Europa y Sudamérica. ${ }^{29}$

El tercer delegado latinoamericano cuyas labores queremos recordar en esta época en Washington, es el joven escritor y diplomático mexicano Matías Romero, quien representó al gobierno republicano de Benito Juárez desde 1861 hasta fines de 1867 en la capital de los Estados Unidos, aunque su labor de "lobista" fue aún más intensa en la ciudad de Nueva York donde pasó mucho tiempo. Resulta interesante que su correspondencia es la más extensa de todos los representantes latinoamericanos del siglo XIX: se han contabilizado más de 17,000 cartas suyas del período 1861-1866, las cuales pueden consultarse - en su mayoría - en el gran archivo de Romero, que se conserva en más de 120 cajas depositadas en las bóvedas de la Biblioteca del Banco de México.

El papel de Romero como agente de propaganda en esta época en los Estados Unidos fue bastante exitoso, logrando despertar sentimientos favorables a la lucha de los republicanos mexicanos en contra de la ocupación militar de México por tropas francesas y austriacas (1863-1867). Además de publicar y distribuir miles de copias de una biografía del presidente Juárez y cientos de folletos que llamaron la atención sobre la difícil situación de México, Romero proporcionó un flujo constante de noticias y artículos informativos a periódicos seleccionados de los Estados Unidos. ${ }^{30}$ Esta labor ayudó a los juaristas en su guerra contra los franceses y contribuyó a impulsar un importante flujo de armas y municiones reenviadas desde Estados Unidos por dos decenas de agentes mexicanos que estuvieron en correspondencia con el ministro Romero. En este entonces, las fuerzas militares de Benito Juárez en el norte de México no contaban con fabricantes de armas, por lo que era esencial importarlos a través de la frontera de Texas. La marina

Donoso, Vicuña Mackenna, p. 218.

Ibid, p. 223

30 Para detalles de sus gestiones diplomáticas ver Silva y Márquez, Matías Romero y el oficio diplomático. 
francesa controlaba las costas de México durante la Intervención y por ello no había posibilidad de obtener armas por mar, y por ello los Estados Unidos eran la fuente principal de material de guerra.

Matías Romero fue bastante lejos en negociar acuerdos con diversos inversores y empresas norteamericanas que esperaba pudieran financiar las fuerzas bajo el mando de Juárez. Inclusive autorizó concesiones de tierras para algunas empresas, incluyendo una denominada la Mexican Mineral and Colonization Company, en la cual el propio ministro tomó algunas acciones y recibió fondos para compras de armas. Al mismo tiempo, algunos de los agentes que trabajaban para Juárez fueron más lejos, como los generales Sánchez Ochoa y Carvajal, quienes emitieron bonos por montos equivalentes a US\$40,000,000 en San Francisco y Nueva York, aunque pronto fueron desautorizados por las autoridades norteamericanas. ${ }^{31}$

Matías Romero se dedicó a cultivar buenas relaciones con amplios círculos de miembros de la élite político del gobierno de la Unión y con directivos de periodistas y empresarios, pero sus esfuerzos por conseguir apoyos oficiales para la causa de Benito Juárez y sus fuerzas tropezaron repetidamente con la renuencia de William Seward, secretario de Estado de Lincoln (y luego del presidente Johnson) a enfrentarse directamente con los franceses. Sin embargo, hacia fines de 1865, los generales de los Estados Unidos, Ulysses S. Grant y Philip Henry Sheridan, pasaron por alto a Seward y comenzaron a apoyar de manera encubierta a Juárez a lo largo de la frontera Texas-México, proporcionando una gran cantidad de armas para las fuerzas militares de Juárez. Para entonces, la intervención en México se había vuelto impopular entre el público francés y era una carga creciente para el tesoro de Francia. Finalmente, el 31 de enero de 1866, Napoleón III ordenó la retirada de las tropas francesas en tres etapas que se sucedieron entre noviembre de 1866 y noviembre de 1867. Seward reconoció que ya no convenía apoyar a Maximiliano y advirtió al Gobierno austriaco contra el reemplazo de las tropas francesas por sus propias fuerzas. Ello condenó a Maximiliano, quien pronto fue derrotado en campo de batalla y fusilado.

Al fin de 1867, con el triunfo de Juárez, Matías Romero regresó a México y pronto fue nombrado secretario de Hacienda y Crédito Público, cargo que desempeñaría tres veces. Más tarde, en los años de 1880, Romero volvió a ser enviado a Washington para representar a su país como embajador (car-

31 La historia algo turbia de estos bonos fue relatado por Matías Romero en sus textos "Responsabilidades contraídas por el Gobierno Nacional de México en los Estados Unidos, en virtud de los contratos celebrados por sus agentes, 1864-1867”, México, Imprenta del Gobierno, 1867, pp. 4-8, <http://cdigital.dgb.uanl.mx/la/1020002896/1020002896_002.pdf>. 
go que ocupó durante dieciocho años), participando muy activamente en la negociación de una serie de acuerdos comerciales entre México y los Estados Unidos. También debe recordarse que Romero fue uno de los promotores de la Primer Conferencia Panamericana, celebrada en Washington en 1889, de la cual fue vicepresidente.

Estados Unidos promovió la realización de esta reunión, la cual sería la primera de muchas otras conocidas como Conferencias Panamericanas. Al mismo tiempo impulsó la creación de la Unión Panamericana, antecedente de la actual Organización de Estados Americanos (OEA). Estos eventos constituyeron un escenario importante para la diplomacia latinoamericana por varios motivos. Puede sugerirse, por una parte, que a partir de esta fecha se amplió notablemente la proyección de muchos diplomáticos, intelectuales y profesionales latinoamericanos, ya que se complementaron las tradicionales gestiones de representación nacionales con una muy intensa participación en foros internacionales desde principios y a lo largo del siglo Xx. Como planteó Marcelo Carmagniani, las prioridades de los Estados en América Latina durante la segunda mitad del siglo XIX fueron el control del territorio interno y la consolidación de los Estados nacionales en términos políticos, económicos, sociales y culturales, lo cual fue complementado por la búsqueda de políticas exteriores que permitieran promover una imagen de modernidad en Europa y Estados Unidos. Para ello fue fundamental su participación en conferencias internacionales, congresos científicos y exposiciones internacionales. ${ }^{32}$

La iniciativa de Estados Unidos al promover las Conferencias Panamericanas desde 1889 era muy diferente a la planteada por Simón Bolívar en el famoso Congreso Anfictiónico de 1826, al mantener silencio sobre el antiguo proyecto político de integración y buscar, por sobre todas las cosas y a través de todos los medios posibles, fomentar los lazos económicos en la región. El panamericanismo tenía una marcada dimensión económica, como uno de los mecanismos para hacerse de información valiosa para promover una mayor cooperación comercial que no escondía su carácter imperialista de penetración. ${ }^{33}$ De hecho, este marcado interés por expandir su economía a través de alcanzar el poder financiero en países débiles fue calificado poco

32 Carmagniani, El otro occidente. América Latina desde la invasión europea hasta la globalización, pp. 194-200.

33 Como menciona Ricardo Salvatore al estudiar este tipo de penetración "los expertos en temas empresarios y los funcionarios de la Unión Panamericana construyeron América Latina como un mercado de inmensas posibilidades, e instaban a los hombres de negocios norteamericanos a prepararse para una grandiosa empresa colectiva: la conquista de esos mercados emergentes". Salvatore. "Panamericanismo práctico. Acerca de la mecánica de penetración comercial norteamericana”, p. 293. 
después como "la diplomacia del dólar", término con el cual Nearing y Freeman, en su libro Dollar Diplomacy (1925), explicaban la interacción de varios actores (inversionistas, banqueros, empresario, políticos, diplomáticos, agentes, aventureros y el ejército), con fines imperialistas. ${ }^{34}$

Durante este tiempo la Unión Panamericana avanzó a ritmo relativamente lento en cuanto a su organización, pues los países latinoamericanos tenían escaso interés y mostraban recelo del "panamericanismo imperial", pero el inicio de la Primera Guerra Mundial generó un cambio en el contexto acercando a estos países a través de un "nuevo panamericanismo" que respondiera a las expectativas que generaba la guerra. Muestra de este giro fue el Congreso Científico Panamericano reunido en Washington pocos meses antes de que Estados Unidos declarara la guerra a Alemania. ${ }^{35}$

Anteriormente ya se habían realizado otros congresos científicos, ${ }^{36}$ pero es evidente que el despegue de este tipo de conferencias se dio a partir de este acercamiento. Para cumplir con esta creciente participación en estos congresos de ciencia, así como en muchos otros que se realizaron sobre temas específicos, se requirió de un número creciente de participantes especializados, por lo que los gobiernos echaron mano de los intelectuales, quienes coyunturalmente se convirtieron en representantes oficiales de las delegaciones ante cada evento. ${ }^{37}$

34 Marichal, "Comentarios sobre un temprano clásico de la izquierda norteamericana: Dollar Diplomacy: A study in American imperialism (1925) por Scott Nearig y Joseph Freeman” p. 327.

35 Desde la Primera Conferencia se creó una Oficina Comercial de las Repúblicas Americanas que recopilaba información y publicaba un boletín, la cual fue re organizada bajo una dirección más reglamentada y fundando la Biblioteca Colón para almacenar la información (1902). En la Conferencia realizada en Rio de Janeiro se decidió continuar con esta Oficina especificando su funcionamiento como estructura permanente para las Conferencias. Fue recién en la Conferencia de 1910 que se llamó Unión Pan-Americana, con sede en Washington y con representantes de los 21 países (excepto Canadá) que tuvieran delegados oficiales en esa ciudad capital. El presidente del Consejo Directivo caería en el Secretario de Estado de Estados Unidos. Las siguientes conferencias durante la década de 1920 flexibilizaron la representación latinoamericana para que pudiera ser cualquier delegado (no sólo los países que tenían representación ante su gobierno como el caso de los gobiernos de México durante la revolución). Sobre la construcción del panamericanismo y la presencia de México remitimos a Carillo, "México en la Unión de las Repúblicas Americanas".

36 El orden es el siguiente: Buenos Aires (1898), Montevideo (1901), Río de Janeiro (1905), Santiago de Chile (1908-1909) y Washington (1915-1916). Esta serie de eventos en particular fue un espacio público para el debate de la ciencia, pero también un espacio de sociabilidad intelectual donde se notaba la tensión de temas que a nivel internacional y regional generaban debate: ciencia y política, conocimiento aplicado y universal, entre otras. Calvo Isaza, "Conocimiento desinteresado y ciencia Americana”.

37 Para ver la variedad de temas tratados en las conferencias panamericanas remitimos a Marichal, México y las conferencias panamericanas. También recomendamos la consulta de la base de datos realizada por El Colegio de México sobre las "Conferencias Internacionales 
Durante las siguientes décadas, la política de la Unión Panamericana si bien mantuvo su preocupación económica tomó un matiz más político en el cual junto a los tratados y acuerdos regionales se desplegó una serie de estrategias para favorecer una dimensión cultural como parte de la diplomacia regional. Así, los intercambios entre norte y sur del continente se sostuvieron gracias a numerosas redes intelectuales que si bien no disolvieron las disputas, se convirtieron en una vía alterna para referirse al avance del panamericanismo.

En este cambio fue importante la impronta de Leo S. Rowe, quien ejerció como director de la Unión Panamericana desde 1920 hasta 1946. Sin dejar de ser un "intelectual imperial", como lo llama Ricardo Salvatore, buscó una interacción entre los intelectuales, fomentando una cooperación intelectual entre las Américas. ${ }^{38}$ Una de estas fructíferas redes, por ejemplo, fue la que alimentó el desarrollo del derecho internacional en la región, el cual fue de especial importancia para el desarrollo del panamericanismo al combinar intereses políticos con académicos, involucrando a todos los países de la región. ${ }^{39}$

Otra variable para entender este giro en el panamericanismo es fruto de la competencia. En 1919 se creó la Sociedad de Naciones y en la década siguiente iniciaron sus actividades diversas instancias de Cooperación Intelectual. América Latina participó activamente de estas instancias porque era un espacio idóneo para establecer un juego estratégico de balance entre los intereses regionales de Estados Unidos y los internacionales de Europa. Además, a través de los intelectuales como representantes de la cultura podían ocupar un nivel menos desfavorecido que otros como el económico. ${ }^{40}$

Algunos de ellos ya habían cumplido funciones diplomáticas y mantuvieron su papel como representantes ante Cooperación Intelectual al mismo tiempo que se desempeñaban como jefes de delegación, embajadores como Alfonso Reyes, Gabriela Mistral, entre muchos otros. ${ }^{41}$ Algunos más se incorporaron a la diplomacia cultural al ser miembros de las Comisiones Nacionales de Cooperación Intelectual para mantener el vínculo entre el organismo internacional, el gobierno nacional y las sociedades letradas.

Americanas, 1889-1936. Recopilación de tratados y otros documentos”, <http://biblio2.colmex.mx/coinam/coinam_1889_1936/base2.htm>.

38 Sobre algunas de sus iniciativas durante su viaje al sur remitimos al trabajo de Salvatore, "Saber hemisférico y disonancias locales".

39 Un análisis sobre cómo se construyó el discurso del derecho internacional en Estados Unidos a inicios del siglo xx y de qué manera impactó en los círculos académicos, políticos e intelectuales de América Latina puede verse en Scarfi, El imperio de la ley.

40 Sobre la relación entre países latinoamericanos y Cooperación Intelectual (especialmente México) véase Pita, Educar para la paz, 2014.

41 Pita, “América (Latina) en París”, 2019. 
También hubo quienes se convirtieron en delegados ante la Sociedad de Naciones (como Isidro Fabela) ${ }^{42}$ o como actores informales, ${ }^{43}$ al ser nombrados correspondientes de esta organización para mantener una relación cercana de los países latinoamericanos. ${ }^{44}$

\section{Cierre}

Para finalizar este ensayo resta invitar al lector a explorar los artículos que componen el presente dossier. Su distribución sigue un orden cronológico, aunque podrá observarse que la mayor parte se concentra en dos grandes períodos: aquél que da cuenta de los inicios de las conferencias panamericanas y el acercamiento diplomático hacia Estados Unidos (en los años de1889-1902, aproximadamente) y el que se desarrolla entre los últimos años de la década de 1920 y mediados de 1940, marcado por el interés de las relaciones bilaterales entre países latinoamericanos, así como entre éstos y Francia. En estos últimos ensayos se abordan cuatro países de América Latina (México, Argentina, Brasil y Uruguay) y dos externos, Estados Unidos y Francia.

El trabajo de Juan Pablo Scarfi es idóneo para empezar el análisis del primer período al abordar la trayectoria de Vicente Quesada, diplomático argentino que se destacó por sus críticas a la Doctrina Monroe. En su lugar, ofreció argumentos sobre la existencia de un derecho internacional latinoamericano basada en el derecho común. Tras analizar cómo construyó Quesada este proyecto, explica su relación con un contexto argentino liberal

42 Sobre la actuación de Fabela ante la SDN véase a Herrera, México y la Sociedad de Naciones, 2014.

43 Son aquellos agentes informales de la diplomacia quienes "sin ser parte de la estructura" tuvieron intervención en el tramado de las relaciones internacionales al influir de alguna manera en la toma de decisiones del Estado. Generalmente, esto se realizó a través del uso del soft power, por ejemplo incidiendo en el clima de opinión pública o en algunas instancias de gobierno para "facilitar una coincidencia de intereses". Argüello y Sánchez, "Introducción”, 2017.

44 A mediados de la década de 1920 la SDN acreditó miembros correspondientes en algunas capitales latinoamericanas para enviar información y mantener una relación cercana con los países miembros de la organización. Esta fue una estrategia para mantener sus membresías, las cuales fueron variables en esa época. Para la SDN estos funcionarios debían actuar como diplomáticos, "un poco francotirador" por su actuar sigiloso, pero no gozaban de las inmunidades". Se esperaba de ellos que siguieran con suma atención e informaran de los acontecimientos nacionales más relevantes (a través de informes ordinarios y extraordinarios) así como del seguimiento puntual de aquello que se relacionara con el estudio y aprobación de convenios y resoluciones. Debían de influir en los debates de opinión pública que se relacionaran con la permanencia del país en la SDN, con una "cuidadosa intervención" de gestión pues no estaban autorizados a tomar iniciativas políticas. Herrera, "Sombras del internacionalismo ginebrino”, pp. 239, 240, 253, 254. 
antiestadounidense, lo cual lleva a afirmar que es fundamental para entender el primer antiimperialismo latinoamericano (que suele sugerirse inicia con la reacción cultural de escritores modernistas en 1898). Basado en lo legal, el proyecto tendría eco entre los juristas y diplomáticos argentinos de las décadas siguientes en Argentina.

Siguiendo con el contexto panamericanista de la Primera Conferencia, Paula Bruno estudia al diplomático argentino Martín García Mérou, quien realizó observaciones sobre el clima de ideas del primer antiimperialismo latinoamericano. Subraya los actores y las dinámicas en las que se desenvolvió la vida diplomática de García y se adentra en los escenarios que marcaron su labor, en este caso, Washington a fines del siglo XIX. La autora recupera los "registros de escritura" del diplomático como una fuente documental rica para entender el contexto de circulación de información, ideas e interpretaciones no sólo del escenario internacional y nacional, sino de su propia definición como diplomáticos a través de la práctica. Esto la lleva a pensar que en este período la diplomacia gozaba de márgenes de autonomía, pues las políticas exteriores no eran rígidas.

Natalia Henrich, por su parte, dedica su artículo a seguir el diálogo entre dos intelectuales y diplomáticos brasileños, Manoel Oliveira Lima y Joaquim Nabuco, con el fin de explicar el contexto en el cual se dio el rompimiento entre ambos y evaluar si éste tuvo que ver o no con su participación en el proceso de americanización de la política exterior brasileña. Para ello pone atención a analizar el libro Pan-americanismo de Oliveira, explicando su contexto de producción (la conferencia panamericana realizada en Río de Janeiro), matizando su antiimperialismo para verlo más como una serie de cuestionamientos que buscaban encontrar una mediación entre la Doctrina Monroe y la Drago. Reconstruye las notas y entrevistas periodísticas publicadas en Brasil para encontrar un tercer personaje destacado de la diplomacia del Brasil, Río Branco, con el fin de mostrar las sutiles diferencias de posturas en torno a un panamericanismo en construcción.

Por otra parte, para dar inicio a los trabajos sobre el segundo período se encuentra el trabajo de Mariana Moraes sobre la relación establecida entre Alfonso Reyes y Luisa Luisi a partir de la acción del Comité UruguayMéxico, ideado entre fines de 1927 y principios de 1928 para dictar una serie de conferencias sobre México. Tras seguir la breve vida del comité, la autora menciona dos tipos de tensiones que merecen ser pensadas. Por una parte, la del intelectual/diplomático que como Reyes, debieron enfrentar críticas del gobierno al considerar que su sentido de diplomacia cultural requería menos veladas literarias y más propaganda política en los medios. Por la otra, la tensión que se produce al interior del campo intelectual al participar de este tipo de acciones de diplomacia cultural, potenciando ciertas rivalidades y competencias. 
Cercano geográficamente, tenemos el artículo de Cecilia Neubauer dedicado a rastrear tras el golpe de estado en Argentina las opiniones que enviaba desde Buenos Aires el diplomático mexicano Rafael Cabrera y en menor medida, las expresiones de preocupación que a su vez se tenían desde el cono sur por los regímenes posrevolucionarios en México. Una idea interesante que deja planteada la autora es que si bien durante el breve período de tiempo que analiza (1930-1931) Cabrera mantuvo las mismas directrices de la diplomacia cultural del embajador predecesor, Alfonso Reyes, el golpe militar generó una nueva coyuntura política que distanció ambos países.

Trasladándonos a París, el texto de Hégron Bastien está dedicado a analizar las gestiones del académico mexicano Luis Chico Goerne, quien tras su renuncia como rector de la UNAM se trasladó a París como consejero de la legación, cargo desde el cual procuró fortalecer los vínculos intelectuales entre México y Francia proponiendo la fundación en París del Instituto Mexicano en 1938. Tras reconstruir esta gestión, el autor menciona que el estudio de esta misión permite plantear que hubo un cambio en la forma de llevar a cabo la diplomacia cultural mexicana al fin del cardenismo, la cual dejó de ser meramente intelectual para convertirse en una gestión más institucional que buscaba conseguir una cooperación política más intensa entre los gobiernos. Aunque no podemos afirmar que esto sea un cambio permanente, consideramos que es una idea sugerente que merecería ser discutida a través de otros estudios de caso.

Como cierre del dossier se cuenta con un artículo de Veremundo Carrillo, el cual nos remite de nueva cuenta a las iniciativas que se dieron desde Estados Unidos para mejorar las relaciones interamericanas. Específicamente, analiza un concurso literario organizado por la Unión Panamericana para calificar la mejor novela latinoamericana, la cual sería traducida y publicada en inglés, pues encuentra en este evento una manera de mostrar de qué manera la historia y la literatura de Las Américas fueron instrumentos válidos de la diplomacia cultural. Para desplegar esto el autor sigue la pista de una persona que estuvo detrás de todo esto: Concha Romero James, jefa de la Oficina de Cooperación Intelectual de la Unión Panamericana, quien supo entretejer con astucia las redes intelectuales y la diplomacia cultural. Aunque el autor no trabaja utilizando la metodología de las redes, reconstruye con claridad el itinerario del concurso señalando las personas, instituciones y revistas involucradas, mostrando la importancia que tiene el estudio de estos eventos aparentemente secundarios en la historia diplomática. 


\section{BIBLIOGRAFÍA}

Ardao, Arturo, América Latina y la Latinidad, México, UNAM, 1993.

Carmagniani, Marcelo, El otro occidente. América Latina desde la invasión europea hasta la globalización. México, El Colegio de México, Fondo de Cultura Económica, 2004.

Calvo Isaza, Óscar, “Conocimiento desinteresado y ciencia Americana. El Congreso Científico (1898-1916)”, Historia Crítica, núm. 45, Bogotá, septiembrediciembre, 2011, pp. 83-113.

Carillo, Veremundo, “México en la Unión de las Repúblicas Americanas. El panamericanismo y la política exterior mexicana, 1889-1942”, tesis para optar por el grado de Doctor en Historia, El Colegio de México, mayo, 2018.

Donoso, Ricardo, Vicuña Mackenna: su vida, escritos y su tiempo, 1831-1886, Santiago-Buenos Aires, Ed. Francisco Aguirre, 1977.

Dumont, Juliette, L'Institute International de Cooperation Intellectuelle et le Brésil (1924-1946), Le Le Peri de la diplomatieculturelle, Paris, L'IHEALCREDAL, 2008.

Granados, Aimer, "Congresos e intelectuales en los inicios de un proyecto y de una conciencia continental latinoamericana, 1826-1860”, Carlos Marichal y Aimer Granados (coords.), Construcción de la identidad latinoamericana. Ensayos de historia intelectual. Siglos XIX y XX, México, El Colegio de México, pp. 39-70.

Herrera, Fabián, “Sombras del internacionalismo ginebrino: los correspondientes en México de la Sociedad de Naciones y de la organización internacional del trabajo”, Ana Rosa Suárez Argüello y Agustín Sánchez Andrés, (coords.), A la sombra de la diplomacia. Actores informales en las relaciones internacional de México, siglos XIX y XX, México, Universidad Michoacana de San Nicolás de Hidalgo, Instituto de Investigaciones Dr. José María Luis Mora, 2017, pp. 239-268.

Herrera, Fabián, México en la Sociedad de Naciones, 1931-1940, México, Secretaría de Relaciones Exteriores, 2014.

Jaksic, Iván, Andrés Bello: Scholarship and Nation-Building in Nineteenth-Century Latin America, Cambridge University Press, 2001.

López-Ocón, Leoncio, Biografía de "La América”: una crónica hispano-americana del liberalismo democrático español (1857-1886), Madrid, CSIC, 1987.

Llorens, Vicente, Liberales y románticos: una emigración española en Inglaterra, 1823-1834, Madrid, Ed. Castalia, 1968.

Lynch, John, Bello y Londres, Caracas, fundación Bello, 1980.

Marichal, Carlos, Historia de la deuda externa de América Latina, Madrid, alianza, 1988. 
_, "El nacimiento de los estudios internacionales sobre América Latina: comentarios a las obras de José María Torres Caicedo y Carlos Calvo a mediados del siglo xIX," Foro Internacional de El Colegio México, julio septiembre, 2015,

$<$ https://forointernacional.colmex.mx/index.php/fi/article/view/2277/2267>.

, "Comentarios sobre un temprano clásico de la izquierda norteamericana: Dollar Diplomacy: A study in American imperialism (1925) por Scott Neargin y Joseph Freeman”, Alexandra Pita y Carlos Marichal, Pensar el antiimperialismo, México, El Colegio de México-Universidad de Colima, 2012, pp. 319-332.

—- México y las conferencias panamericanas, 1889-1938. Antecedentes de la globalización, México, Secretaría de Relaciones Exteriores, 2002.

Myers, Jorge, "El letrado patriota: los hombres de letras hispanoamericanos en la encrucijada del colapso del imperio español en América” en Carlos Altamirano (ed.), Historia de los intelectuales en América Latina, vol. 1, Buenos Aires, Katz Ediciones, pp. 121-144.

Pita, Alexandra, "La diplomacia cultural mexicana en la entreguerras: una aproximación al debate”, Herrera Fabián (coord.), Diplomacia oficiosa, representaciones y redes extraoficiales en la historia de América Latina: un homenaje a la trayectoria académica de Salvador E. Morales Pérez (1939-2012), México, Universidad Michoacana de San Nicolás de Hidalgo, Instituto de Historia de Cuba y Universidad Católica Andrés Bello-Caracas, 2015, pp. 107-130.

Pita, Alexandra, Educar para la paz. México y la Cooperación Intelectual Internacional, 1922-1948, México, Secretaría de Relaciones Exteriores-Universidad de Colima, 2014.

, “América (Latina) en París. Mistral, Reyes y Torres Bodet en la colección iberoamericana, 1927-1940”, en Fabián Herrera León y Yannick Wehrli (coords.), América Latina y el internacionalismo ginebrino de entreguerras: resonancias e implicaciones, México, Dirección General del Acervo Histórico Diplomático, 2019.

Rodríguez O., Jaime, El nacimiento de Hispanoamérica: Vicente Rocafuerte y el hispanoamericanismo, 1808-1832, México, Fondo de Cultura Económica, 1980.

Rojas, Rafael, "Traductores de la libertad; el americanismo de los primeros republicanos”, en Altamirano, Carlos (ed.), Historia de los intelectuales en América Latina, Katz, vol. 1, 2010, pp. 205-226.

Sablonierre, Catherine, El Correo de Ultramar (1842-1886) y la ciencia: entre labor educativa y propaganda política, $<$ https://www.academia.edu/8629881/el_correo_de_ultramar_y_la_ciencia>.

Salvatore, Ricardo, "Panamericanismo práctico. Acerca de la mecánica de penetración comercial norteamericana”, Ricardo D. Salvatore (ed.), Culturas impe- 
riales. Experiencia y representación en América, Asia y África, Rosario, Beatriz Viterbo, 2005, pp. 269-300.

-, "Saber hemisférico y disonancias locales. Leo S. Rowe en Argentina, 19061019”, Ricardo Salvatore (comp.), Los lugares del saber, contextos locales y redes trasnacionales en la formación del conocimiento moderno, Rosario: Beatriz Viterbo, 2007, pp. 327-367.

Scarfi, Juan Pablo, “Introducción”, dossier, diplomacia, vida cultural y circulación de saberes Programa interuniversitario de historia política, dossier número 66, noviembre, 2015, <http://historiapolitica.com/dossiers/diplomaciacirculacion-saberes/>.

— El imperio de la ley. James Brown Scott y la construcción de un orden jurídico interamericano. Buenos Aires, FCE, 2014.

Silva, Sergio y Márquez, Graciela, Matías Romero y el oficio diplomático, 18371898, México, Instituto Matías Romero, 2016.

Suárez Argüello, Ana Rosa y Sánchez Andrés, Agustín, “Introducción”, Ana Rosa Suárez Argüello y Agustín Sánchez Andrés (coords.), A la sombra de la diplomacia. Actores informales en las relaciones internacionales de México, siglos XIX Y XX, México, Universidad Michoacana de San Nicolás de HidalgoInstituto de Investigaciones Dr. José María Luis Mora, 2017, pp. 9-10.

Véliz, Claudio, “'Egaña’, Lambert and the Cjilean Minning Association of 1825”, Hispanic American Historical Review, vol. 55, no. 4, 1975, pp. 645-647. 